\title{
A CHARACTERIZATION OF COLLECTIONS OF TWO-POINT SETS WITH THE UNIQUENESS PROPERTY
}

\author{
TAKASHI OKAMOTO AND MANABU SHIROSAKI
}

(Received December 12, 2003, revised October 29, 2004)

\begin{abstract}
We give a sufficient condition for a collection of two-point sets to have the uniqueness property for meromorphic functions.
\end{abstract}

1. Introduction. For nonconstant meromorphic (or entire) functions $f$ and $g$ on $\boldsymbol{C}$ and a discrete set $S$ in $\hat{\boldsymbol{C}}=\boldsymbol{C} \cup\{\infty\}$ (or $\boldsymbol{C}$ ), we write $f^{*}(S)=g^{*}(S)$ if $f^{-1}(S)=g^{-1}(S)$ and if for each $z_{0} \in f^{-1}(S)$ two functions $f-f\left(z_{0}\right)$ and $g-g\left(z_{0}\right)$ have a zero point with the same multiplicity at $z_{0}$, where a zero point of $f-\infty$ and $g-\infty$ means that of $1 / f$ and $1 / g$, respectively.

Let $\left\{S_{1}, \ldots, S_{q}\right\}$ be a finite collection of pairwise disjoint discrete sets in $\hat{\boldsymbol{C}}$ (or $\boldsymbol{C}$ ). If $f^{*}\left(S_{j}\right)=g^{*}\left(S_{j}\right)(1 \leq j \leq q)$ imply $f=g$ for two nonconstant meromorphic functions $f$ and $g$ (or two nonconstant entire functions $f$ and $g$ ) on $\boldsymbol{C}$, then the collection is said to have the uniqueness property for meromorphic (or entire) functions (abbreviated to UPM (or UPE)). As examples of such collections, we know Nevanlinna's four values theorem ([N]), uniqueness range sets for meromorphic or entire functions ([LY], [Y2]) and so on ([S], [Y3]). However, there is no result except $[\mathrm{N}],[\mathrm{T}$, Theorem 1.4] and [Y1] that characterizes such collections. In this paper we give a characterization for collections of two-point sets with the uniqueness property.

The authors would like to thank the referee for pertinent comments.

2. Combinatorial lemmas and Borel's lemma. For a large part of this section we proceed as in [F, §2]. Let $G$ be a torsion-free abelian multiplicative group, and consider a $q$-tuple $A=\left(a_{1}, a_{2}, \ldots, a_{q}\right)$ of elements $a_{i}$ in $G$. For a subgroup $\tilde{A}$ of $G$ generated by $a_{1}, a_{2}, \ldots, a_{q}$, we can take a basis $\left\{b_{1}, \ldots, b_{t}\right\}$ of $\tilde{A}$. Then each $a_{i}$ can be uniquely represented as

$$
a_{i}=b_{1}{ }^{l_{i 1}} b_{2}{ }^{l_{i 2}} \cdots b_{t}{ }^{l_{i t}}
$$

with suitable integers $l_{i \tau}$.

DEFinition 2.1. We call integers $p_{1}, p_{2}, \ldots, p_{t}$ with the following property to be generic with respect to $l_{i \tau}$ and call the integers $l_{i}:=\sum_{\tau=1}^{t} l_{i \tau} p_{\tau}$ representations of $a_{i}(1 \leq$ $i \leq q)$ :

$$
\text { if } l_{i}= \pm l_{j}, \text { then }\left(l_{i 1}, l_{i 2}, \ldots, l_{i t}\right)= \pm\left(l_{j 1}, l_{j 2}, \ldots, l_{j t}\right),
$$

2000 Mathematics Subject Classification. Primary 30D35.

Key words and phrases. Uniqueness theorem, Nevanlinna theory. 
where double signs are in the same order.

For example, it is enough to take $p_{\tau}=p^{\tau-1}(1 \leq \tau \leq t)$ for an integer $p>$ $2 \max \left\{\left|l_{i \tau}\right| ; 1 \leq i \leq q, 1 \leq \tau \leq t\right\}$.

DEFINITION 2.2. Let $q \geq r>s \geq 1$ and $A=\left(a_{1}, a_{2}, \ldots, a_{q}\right)$ a $q$-tuple of elements $a_{i}$ in $G$.

(i) We call $A$ to have the property $\left(P_{r, s}\right)$ if any $r$ elements $a_{l(1)}, a_{l(2)}, \ldots, a_{l(r)}$ in $A$ satisfy the condition that, for any given $i_{1}, i_{2}, \ldots, i_{s}\left(1 \leq i_{1}<\cdots<i_{s} \leq r\right)$, there exist some other $j_{1}, j_{2}, \ldots, j_{s}\left(1 \leq j_{1}<\cdots<j_{s} \leq r,\left\{i_{1}, \ldots, i_{s}\right\} \neq\left\{j_{1}, \ldots, j_{s}\right\}\right)$ satisfying

$$
a_{l\left(i_{1}\right)} a_{l\left(i_{2}\right)} \cdots a_{l\left(i_{s}\right)}=a_{\iota\left(j_{1}\right)} a_{l\left(j_{2}\right)} \cdots a_{\iota\left(j_{s}\right)} .
$$

(ii) We call $A$ to have the property $\left(P^{*}\right)$ if each element of $\left\{\prod_{j=1}^{q} a_{j} \varepsilon_{j} ; \varepsilon_{j}=0,1(1 \leq\right.$ $j \leq q)$ \} coinsides with another one of this set, where $a_{j}{ }^{0}=1$ (the unit element of $G$ ).

Let us study relations among $a_{i}$ for a $q$-tuple $a=\left(a_{1}, a_{2}, \ldots, a_{q}\right)$ with the property $\left(P_{r, s}\right)$ or $\left(P^{*}\right)$. To this end, we take representations $l_{1}, l_{2}, \ldots, l_{q}$ of $a_{1}, a_{2}, \ldots, a_{q}$ for suitably chosen basis and generic integers. We arrange the order of representations to be

$$
l_{j_{1}} \leq l_{j_{2}} \leq \cdots \leq l_{j_{q}} .
$$

LEMMA 2.3 (for the proof, see [F]). If a q-tuple has the property $\left(P_{r, s}\right)$, it holds that

$$
l_{j_{s}}=l_{j_{s+1}}=\cdots=l_{j_{s+u}}
$$

and hence

for $u:=q-r+1$.

$$
a_{j_{s}}=a_{j_{s+1}}=\cdots=a_{j_{s+u}}
$$

LEMMA 2.4. If a q-tuple $\left(a_{1}, a_{2}, \ldots, a_{q}\right)$ has the property $\left(P^{*}\right)$, then at least one $a_{j}$ is the unit element 1 .

Proof. Assume that none of $a_{1}, a_{2}, \ldots, a_{q}$ is the unit element. Then $l_{j} \neq 0, j=$ $1,2, \ldots, q$. Let $m$ be the integer such that

$$
l_{j_{1}} \leq l_{j_{2}} \leq \cdots \leq l_{j_{m}}<0<l_{j_{m+1}} \leq \cdots \leq l_{j_{q}} .
$$

If $m=0$ or $m=q$, then there is no negative $l_{j}$ or positive $l_{j}$, respectively. Consider the case of $m>0$. Then $l_{j_{1}}+l_{j_{2}}+\cdots+l_{j_{m}}$ is the minimum partial sum of representations and there is no other such combination with this sum, which contradicts the assumption that $\left(a_{1}, a_{2}, \ldots, a_{q}\right)$ has the property $\left(P^{*}\right)$. Hence we have $m=0$. In the case of $m=0$, we get a contradiction by the same method. In consequence, there is some $l_{j}=0(1 \leq j \leq q)$.

In the next section we investigate the torsion-free abelian multiplicative group $G=\mathcal{E} / \mathcal{C}$, where $\mathcal{E}$ is the abelian group of entire functions without zeros and $\mathcal{C}$ is the subgroup of nonzero constant functions.

We close this section by the following Borel's Lemma, whose proof can be found, for example, on p. 186 of [L]. 
LEMMA 2.5. If entire functions $\alpha_{0}, \alpha_{1}, \ldots, \alpha_{n}$ without zeros satisfy

$$
\alpha_{0}+\alpha_{1}+\cdots+\alpha_{n}=0
$$

then for each $j=0,1, \ldots, n$ there exists some $k \neq j$ such that $\alpha_{j} / \alpha_{k}$ is constant.

\section{Main theorems.}

THEOREM 3.1. Let $\left\{S_{1}, \ldots, S_{q}\right\}$ be a collection of pairwise disjoint two-point sets. Put $S_{j}=\left\{\xi_{j}, \eta_{j}\right\}=\left\{z ; z^{2}+a_{j} z+b_{j}=0\right\}\left(\xi_{j} \neq \eta_{j}\right)$. Assume that there is no Möbius transformation $T$ such that $T\left(\xi_{j}\right)=\eta_{j}$ and $T\left(\eta_{j}\right)=\xi_{j}$ for three distinct $j$ 's. If $q \geq 6$, then the collection has UPM.

LEMmA 3.2. Let $S_{j}=\left\{\xi_{j}, \eta_{j}\right\}=\left\{z ; z^{2}+a_{j} z+b_{j}=0\right\}(j=1,2,3)$ be pairwise disjoint. Then there exists a Möbius transformation $T$ such that $T\left(\xi_{j}\right)=\eta_{j}$ and $T\left(\eta_{j}\right)=$ $\xi_{j}(j=1,2,3)$ if and only if

$$
\left|\begin{array}{lll}
1 & a_{1} & b_{1} \\
1 & a_{2} & b_{2} \\
1 & a_{3} & b_{3}
\end{array}\right|=0 .
$$

Proof. Assume that (3.3) holds. Then there exist constants $a, b, c$ such that $(a, b, c) \neq$ $(0,0,0)$ and $a+b\left(\xi_{j}+\eta_{j}\right)+c \xi_{j} \eta_{j}=0$ for $j=1,2,3$. We can take the Möbius transformation $T(z)=-(b z+a) /(c z+b)$ satisfying $T\left(\xi_{j}\right)=\eta_{j}, T\left(\eta_{j}\right)=\xi_{j}$ for $j=1,2,3$.

Conversely, assume that a Möbius transformation $T(z)=(a z+b) /(c z+d)$ satisfies $T\left(\xi_{j}\right)=\eta_{j}, T\left(\eta_{j}\right)=\xi_{j}$ for $j=1,2,3$. Then we have $b+a \xi_{j}-d \eta_{j}-c \xi_{j} \eta_{j}=0$ and $b+a \eta_{j}-d \xi_{j}-c \xi_{j} \eta_{j}=0$ for $j=1,2,3$. By adding these identities, we get $2 b+(a-d)\left(\xi_{j}+\right.$ $\left.\eta_{j}\right)-2 c \xi_{j} \eta_{j}=0$ for $j=1,2,3$. Then (3.3) follows from these, since $b=c=a-d=0$ is impossible.

Proof of TheOREM 3.1. It suffices to treat the case of $q=6$. Assume that $f^{*}\left(S_{j}\right)=$ $g^{*}\left(S_{j}\right)$ for two nonconstant meromorphic functions $f$ and $g$. We may write $f=f_{1} / f_{0}$ by entire functions $f_{0}, f_{1}$ without common zeors, and $g=g_{1} / g_{0}$ in a similar manner. Then there are entire functions $\alpha_{j}$ without zeros such that

$$
f_{1}^{2}+a_{j} f_{1} f_{0}+b_{j} f_{0}^{2}=\alpha_{j}\left(g_{1}^{2}+a_{j} g_{1} g_{0}+b_{j} g_{0}^{2}\right), \quad j=1, \ldots, 6
$$

by the assumption $f^{*}\left(S_{j}\right)=g^{*}\left(S_{j}\right)$. These are expressed as

$$
\left(\begin{array}{cccccc}
1 & a_{1} & b_{1} & \alpha_{1} & a_{1} \alpha_{1} & b_{1} \alpha_{1} \\
1 & a_{2} & b_{2} & \alpha_{2} & a_{2} \alpha_{2} & b_{2} \alpha_{2} \\
\vdots & \vdots & \vdots & \vdots & \vdots & \vdots \\
1 & a_{6} & b_{6} & \alpha_{6} & a_{6} \alpha_{6} & b_{6} \alpha_{6}
\end{array}\right)\left(\begin{array}{c}
f_{1}^{2} \\
f_{1} f_{0} \\
f_{0}^{2} \\
-g_{1}^{2} \\
-g_{1} g_{0} \\
-g_{0}^{2}
\end{array}\right)=\left(\begin{array}{c}
0 \\
0 \\
\vdots \\
0
\end{array}\right)
$$


where the determinant of the square matrix is identically zero because of $f_{1} \not \equiv 0$. In the expansion of the determinant, the coefficient of $\alpha_{j_{1}} \alpha_{j_{2}} \alpha_{j_{3}}$ is given by

$$
\pm\left|\begin{array}{lll}
1 & a_{j_{1}} & b_{j_{1}} \\
1 & a_{j_{2}} & b_{j_{2}} \\
1 & a_{j_{3}} & b_{j_{3}}
\end{array}\right| \cdot\left|\begin{array}{ccc}
1 & a_{k_{1}} & b_{k_{1}} \\
1 & a_{k_{2}} & b_{k_{2}} \\
1 & a_{k_{3}} & b_{k_{3}}
\end{array}\right|,
$$

where $\left\{j_{1}, j_{2}, j_{3}, k_{1}, k_{2}, k_{3}\right\}=\{1,2, \ldots, 6\}$. These coefficients are not zero by the assumption together with Lemma 3.2. Also, by using Borel's Lemma for each triple $\left(j_{1}, j_{2}, j_{3}\right)$, there exists another triple $\left(k_{1}, k_{2}, k_{2}\right)$ such that $\left(\alpha_{j_{1}} \alpha_{j_{2}} \alpha_{j_{3}}\right) /\left(\alpha_{k_{1}} \alpha_{k_{2}} \alpha_{k_{3}}\right)$ is constant, where $1 \leq j_{1}<j_{2}<j_{3} \leq 6,1 \leq k_{1}<k_{2}<k_{3} \leq 6$ and $\left\{j_{1}, j_{2}, j_{3}\right\} \neq\left\{k_{1}, k_{2}, k_{3}\right\}$. From Lemma 2.3 we can deduce that there exist $j_{1}, j_{2}, 1 \leq j_{1}<j_{2} \leq 6$, such that $\alpha_{j_{1}} / \alpha_{j_{2}}$ is constant. Without loss of generality, we may assume that $j_{1}=1, j_{2}=2$. Put $c=\alpha_{1} / \alpha_{2}$ and define a rational function $\varphi\left(z_{0}, z_{1}\right)$ by

$$
\varphi\left(z_{0}, z_{1}\right)=\frac{z_{1}^{2}+a_{1} z_{1} z_{0}+b_{1} z_{0}^{2}}{z_{1}^{2}+a_{2} z_{1} z_{0}+b_{2} z_{0}^{2}} .
$$

Then we have $\varphi\left(f_{0}, f_{1}\right)=c \varphi\left(g_{0}, g_{1}\right)$, i.e.,

$$
\frac{f^{2}+a_{1} f+b_{1}}{f^{2}+a_{2} f+b_{2}}=c \frac{g^{2}+a_{1} g+b_{1}}{g^{2}+a_{2} g+b_{2}} .
$$

(i) The case of $c=1$. In this case, we get from (3.5)

$$
(f-g)\left\{\left(a_{1}-a_{2}\right) f g+\left(b_{1}-b_{2}\right)(f+g)+\left(a_{2} b_{1}-a_{1} b_{2}\right)\right\}=0 .
$$

Assume that $f \neq g$. Then we have

$$
g=-\frac{\left(b_{1}-b_{2}\right) f+\left(a_{2} b_{1}-a_{1} b_{2}\right)}{\left(a_{1}-a_{2}\right) f+\left(b_{1}-b_{2}\right)} .
$$

Note that $a_{1}=a_{2}$ and $b_{1}=b_{2}$ imply $S_{1}=S_{2}$, which does not occur.

Now we consider the Möbius transformation

$$
T(z)=-\frac{\left(b_{1}-b_{2}\right) z+\left(a_{2} b_{1}-a_{1} b_{2}\right)}{\left(a_{1}-a_{2}\right) z+\left(b_{1}-b_{2}\right)},
$$

which exchanges $\xi_{j}$ with $\eta_{j}$ for $j=1,2$, and note that $T^{-1}(z)=T(z)$. If $f\left(z_{0}\right)=\xi_{j}$ for some $j, 3 \leq j \leq 6$, then by the assumption $g\left(z_{0}\right)=\xi_{j}$ or $g\left(z_{0}\right)=\eta_{j}$. However, the latter implies that $T$ exchanges $\xi_{j}$ with $\eta_{j}$, which contradicts the assumption. The former implies that $\xi_{j}$ is a fixed point of $T$. Since $T$ has at most two fixed points, at least six points of $\xi_{3}, \eta_{3}, \ldots, \xi_{6}, \eta_{6}$ are Picard exceptional values of $f$, which is also a contradiction. In consequence, $f=g$ in this case.

(ii) The case of $c \neq 1$. If there is a point $z_{0}$ such that $f\left(z_{0}\right)=g\left(z_{0}\right) \notin S_{1} \cup S_{2}$, then $c=1$ by (3.5). Therefore $f^{-1}\left(\xi_{j}\right)=g^{-1}\left(\eta_{j}\right)$ and $f^{-1}\left(\eta_{j}\right)=g^{-1}\left(\xi_{j}\right)$ for $j=3, \ldots, 6$. Also, there exist entire functions $\beta_{j}, \gamma_{j}$ without zeros such that

$$
f_{1}-\xi_{j} f_{0}=\beta_{j}\left(g_{1}-\eta_{j} g_{0}\right), \quad g_{1}-\xi_{j} g_{0}=\gamma_{j}\left(f_{1}-\eta_{j} f_{0}\right), \quad j=3, \ldots, 6 .
$$


By multiplying each side of these equations, we have

$$
f_{1} g_{1}-\xi_{j}\left(f_{1} g_{0}+f_{0} g_{1}\right)+\xi_{j}^{2} f_{0} g_{0}=\delta_{j}\left\{f_{1} g_{1}-\eta_{j}\left(f_{1} g_{0}+f_{0} g_{1}\right)+\eta_{j}^{2} f_{0} g_{0}\right\}
$$

where $\delta_{j}=\beta_{j} \gamma_{j}$. Since these identities yields the equation

$$
\left(\begin{array}{ccc}
1-\delta_{3} & \xi_{3}-\eta_{3} \delta_{3} & \xi_{3}^{2}-\eta_{3}^{2} \delta_{3} \\
1-\delta_{4} & \xi_{4}-\eta_{4} \delta_{4} & \xi_{4}^{2}-\eta_{4}{ }^{2} \delta_{4} \\
1-\delta_{5} & \xi_{5}-\eta_{5} \delta_{5} & \xi_{5}^{2}-\eta_{5}{ }^{2} \delta_{5}
\end{array}\right)\left(\begin{array}{c}
f g \\
-(f+g) \\
1
\end{array}\right)=\left(\begin{array}{l}
0 \\
0 \\
0
\end{array}\right),
$$

we have

$$
\left|\begin{array}{lll}
1-\delta_{3} & \xi_{3}-\eta_{3} \delta_{3} & \xi_{3}^{2}-\eta_{3}^{2} \delta_{3} \\
1-\delta_{4} & \xi_{4}-\eta_{4} \delta_{4} & \xi_{4}^{2}-\eta_{4}^{2} \delta_{4} \\
1-\delta_{5} & \xi_{5}-\eta_{5} \delta_{5} & \xi_{5}^{2}-\eta_{5}^{2} \delta_{5}
\end{array}\right| \equiv 0
$$

In the expansion of the determinant, none of the coefficients of each element of $B=\left\{1, \delta_{3}, \delta_{4}\right.$, $\left.\delta_{5}, \delta_{3} \delta_{4}, \delta_{4} \delta_{5}, \delta_{3} \delta_{5}, \delta_{3} \delta_{4} \delta_{5}\right\}$ is zero. Then each of these eight functions has a partner among them such that their ratio is constant by Borel's Lemma. In other words, $C=\left(\left[\delta_{3}\right],\left[\delta_{4}\right],\left[\delta_{5}\right]\right)$ has the property $\left(P^{*}\right)$, where for $\varphi \in \mathcal{E}$ we express by $[\varphi]$ the class in $\mathcal{E} / \mathcal{C}$ to which $\varphi$ belongs. Indeed, a product of any three elements in $C$ coincides with some element in $B$ up to multiplying constant, and also it is proportional to another element in $B$. However the latter coincides with a product of different at most three elements in $C$ or the unit element from the beginning. Thus $C$ has the property $\left(P^{*}\right)$. It follows from Lemma 2.4 that there exists $j_{3}, 3 \leq j_{3} \leq 5$, such that $\delta_{j_{3}}$ is constant, and we may assume that $j_{3}=3$. Put $d=\delta_{3}$. Then we have

$$
f_{1} g_{1}-\xi_{3}\left(f_{1} g_{0}+f_{0} g_{1}\right)+\xi_{3}^{2} f_{0} g_{0}=d\left\{f_{1} g_{1}-\eta_{3}\left(f_{1} g_{0}+f_{0} g_{1}\right)+\eta_{3}^{2} f_{0} g_{0}\right\}
$$

and

$$
f g-\xi_{3}(f+g)+\xi_{3}^{2}=d\left\{f g-\eta_{3}(f+g)+\eta_{3}{ }^{2}\right\}
$$

Without loss of generality, we may assume that $f$ takes at least three values of $\xi_{4}, \eta_{4}, \xi_{5}, \eta_{5}$ by assuming that an exceptional value belongs to $S_{6}$, if it exists in $S_{4} \cup S_{5} \cup S_{6}$. Hence we have

$$
\xi_{j} \eta_{j}-\xi_{3}\left(\xi_{j}+\eta_{j}\right)+\xi_{3}^{2}=d\left\{\xi_{j} \eta_{j}-\eta_{3}\left(\xi_{j}+\eta_{j}\right)+\eta_{3}{ }^{2}\right\}, \quad j=4,5
$$

and

$$
\xi_{3}^{2}+a_{j} \xi_{3}+b_{j}=d\left\{\eta_{3}^{2}+a_{j} \eta_{3}+b_{j}\right\}, \quad j=4,5 .
$$

By expressing these as

$$
\left(\begin{array}{cc}
\xi_{3}^{2}+a_{4} \xi_{3}+b_{4} & \eta_{3}^{2}+a_{4} \eta_{3}+b_{4} \\
\xi_{3}^{2}+a_{5} \xi_{3}+b_{5} & \eta_{3}^{2}+a_{5} \eta_{3}+b_{5}
\end{array}\right)\left(\begin{array}{c}
1 \\
-d
\end{array}\right)=\left(\begin{array}{l}
0 \\
0
\end{array}\right),
$$

we have

$$
\left|\begin{array}{ll}
\xi_{3}^{2}+a_{4} \xi_{3}+b_{4} & \eta_{3}^{2}+a_{4} \eta_{3}+b_{4} \\
\xi_{3}^{2}+a_{5} \xi_{3}+b_{5} & \eta_{3}^{2}+a_{5} \eta_{3}+b_{5}
\end{array}\right|=0
$$


On the other hand, we see that

$$
\left|\begin{array}{ll}
\xi_{3}^{2}+a_{4} \xi_{3}+b_{4} & \eta_{3}^{2}+a_{4} \eta_{3}+b_{4} \\
\xi_{3}{ }^{2}+a_{5} \xi_{3}+b_{5} & \eta_{3}^{2}+a_{5} \eta_{3}+b_{5}
\end{array}\right|=\left|\begin{array}{ccc}
1 & a_{3} & b_{3} \\
1 & a_{4} & b_{4} \\
1 & a_{5} & b_{5}
\end{array}\right| \cdot\left|\begin{array}{ccc}
\xi_{3}^{2} & \eta_{3}^{2} & 1 \\
\xi_{3} & \eta_{3} & 0 \\
1 & 1 & 0
\end{array}\right| \neq 0,
$$

which is a contradiction. This completes the proof.

THEOREM 3.6. Let $\left\{S_{1}, \ldots, S_{q}\right\}$ be a collection of pairwise disjoint two-point sets. Put $S_{j}=\left\{\xi_{j}, \eta_{j}\right\}=\left\{z ; z^{2}+a_{j} z+b_{j}=0\right\}\left(\xi_{j} \neq \eta_{j}\right)$. Assume that there is no Möbius transformation $T$ such that $T\left(\xi_{j}\right)=\eta_{j}$ and $T\left(\eta_{j}\right)=\xi_{j}$ for three distinct $j$ 's and that $a_{j} \neq a_{k}(j \neq k)$. If $q \geq 5$, then the collection has $U P E$.

The outline of the proof is the same as that of the proof of Theorem 3.1. There exist two points, which are contained in the sixth set $S_{6}$. The first is the point to get (3.4) and the second is the one to get (3.6), where we assumed that one of the exceptional values of $f$ is in $S_{6}$ if they exist in $S_{4} \cup S_{5} \cup S_{6}$. We can avoid the second, since entire functions have no pole.

Instead of (3.4) we can use the relation

$$
\left(\begin{array}{cccccc}
0 & 0 & 1 & 0 & 0 & 1 \\
1 & a_{1} & b_{1} & \alpha_{1} & a_{1} \alpha_{1} & b_{1} \alpha_{1} \\
\vdots & \vdots & \vdots & \vdots & \vdots & \vdots \\
1 & a_{5} & b_{5} & \alpha_{5} & a_{5} \alpha_{5} & b_{5} \alpha_{5}
\end{array}\right)\left(\begin{array}{c}
f^{2} \\
f \\
1 \\
-g^{2} \\
-g \\
-1
\end{array}\right)=\left(\begin{array}{c}
0 \\
0 \\
\vdots \\
0
\end{array}\right)
$$

where the determinant of the square matrix is identically zero. Also, we see by the assumptions that in its expansion none of the coefficients of $\alpha_{j_{1}} \alpha_{j_{2}} \alpha_{j_{3}}, 0 \leq j_{1}<j_{2}<j_{3} \leq 5$, is zero, where $\alpha_{0} \equiv 1$. Then there exist $j_{1}, j_{2}, 0 \leq j_{1}<j_{2} \leq 5$, such that $\alpha_{j_{1}} / \alpha_{j_{2}}$ is constant. If $j_{1} \geq 1$, then the rest of the proof proceeds in the same way as that of Theorem 3.1.

Now we consider the case of $j_{1}=0$. We may assume that $j_{2}=1$. Put $c:=\alpha_{1}$ (constant). Then we have

$$
f^{2}+a_{1} f+b_{1}=c\left(g^{2}+a_{1} g+b_{1}\right) .
$$

If $c=1$ and $f \neq g$, then $f+g+a_{1}=0$. We may assume that neither of $\xi_{2}$ and $\eta_{2}$ are exceptional values of $f$ and $g$. If there is a point $z_{0}$ such that $f\left(z_{0}\right)=\xi_{2}, g\left(z_{0}\right)=\eta_{2}$, then we get $a_{1}=a_{2}$, which is a contradiction. Otherwise, we have $2 \xi_{2}=-a_{1}=2 \eta_{2}$, which is also a contradiction. Consequently, $f=g$ if $c=1$.

When $c \neq 1$, we can proceed in the same way as in the proof of the case (ii) of Theorem 3.1 , and complete the proof.

\section{REFERENCES}

[F] H. Fujimoto, The uniqueness problem of meromorphic maps into the complex projective space, Nagoya Math. J. 58 (1975), 1-23.

[L] S. LANG, Introduction to complex hyperbolic spaces, Springer-Verlag, New York, 1987.

[LY] P. LI AND C.-C. YANG, Some further results on the unique range sets of meromorphic functions, Kodai Math. J. 18 (1995), 437-450. 
[N] R. Nevanlinna, Einige Eindeutigkeitssätze in der Theorie der meromorphen Funktionen, Acta Math. 48 (1926), 367-391.

[S] M. ShIRosaki, On polynomials which determine holomorphic mappings, J. Math. Soc. Japan 49 (1997), 289-298.

[T] K. ToHgE, Meromorphic functions covering certain finite sets at the same points, Kodai Math. J. 11 (1988), 249-279.

[Y1] H.-X. YI, On the uniqueness of meromorphic functions, Acta Math. Sinica 31 (1988), 570-576

[Y2] H.-X. YI, Unicity theorems for entire functions, Kodai Math. J. 17 (1994), 133-141.

[Y3] H.-X. YI, A question of Gross and the uniqueness of entire functions, Nagoya Math. J. 138 (1995), $169-177$.

SEIBo HighsCOOL

КYОTO 612-0878

JAPAN

\author{
Department of Mathematical Sciences \\ COLLEGE OF ENGINEERING \\ Osaka Prefecture University \\ SAKAI 599-8531 \\ JAPAN \\ E-mail address: mshiro@ms.osakafu-u.ac.jp
}

